When forward displacement of lens and iris are found denoting that the obstruction lies at the lento-zonular level, a preliminary posterior sclerotomy to permit of their retraction, as advised by Priestley Smith, suggests itself as a suitable procedure, to be immediately followed by iridectomy, sclerotomy, or one of their modifications. The object of these operations is to produce a permanent path of exit for the intraocular fluids, either by opening up the normal filtration angle, and so removing the iris as to prevent the possibility of the recurrence of the closure, or, where this is impracticable, to provide a sufficient substitute.

We know that in the earlier periods of glaucoma the iris is in contact with, but not adherent to, the corneal periphery, and cure may be effected, as has been pointed out by Treacher Collins, by the mere escape of aqueous or by the dragging on the iris during its removal; but iridectomy should have in view much more than this. As frequently or generally performed, the iris is seized by forceps and then cut off, with the result that sufficient remains to again block up the exit. The better way is to tear the iris from its junction with the ciliary body, where it is flimsiest, from one end of the wound up to the other, and failure to do this has been the most prolific cause of non-success. Indeed, in forming an opinion as to the benefits of iridectomy one should be careful to distinguish such operations from those in which the iris is merely snipped away.

When iris and cornea are so adherent that they can not be separated, i. e., in chronic, long-continued cases, our hope lies in the formation of subconjunctival fistulæ. When these are permanent and successful they are lined by atrophic iris which keeps the wound from healing. Where this adhesion is known to exist an opening into the posterior chamber through the iritic root as advocated by Treacher Collins, Nicati and Knies, has the best prospect of success, and the V-shaped incision of Nicati, which also freely divides the base of the iris, but upon which I can not speak from personal experience, appears to be attended by excellent results. In those cases in which the actual position of the angle may at first be doubtful, and in which an iridectomy proves useless, Nicati's incision downward might well replace the secondary sclerotomy so frequently employed.

Vincentiis of Naples claims for his operation of tearing or cutting away the iris from the cornea with a sickle-shaped needle that, when these tissues are adherent it acts in a manner superior to either iridectomy or sclerotomy.

In concluding this question I would advocate the earliest possible operation in practically all cases of primary glaucoma (at the same time that attention is given to the general health) independent of contraction of the field, and so long as there is any vision to be saved or pain to be relieved; and that the better eye should first be treated, because in it there is more to lose by delay, and because in the event of only moderate success, or worse, with the first eye, the patient may be disinclined to return on account of the second.

Question 4.--It has been stated by ophthalmic surgeons of the highest order, among whom is one who has done some of the best work in recent ocular pathology, that the cataracts rapidly following upon iridectomy are always due to a wound of the capsule of the lens received in the course of operation. That the possibility of this is one of the serious objections to operation for glaucoma, that it may readily happen in the hands of the most skillful, especially when the cornea is small and the anterior chamber shallow, and that the wound may arise not only from the edge of the knife, but also from the back of a "Graefe," from the iridectorny forceps, or during reposition of the edges of the coloboma, will, I think, be readily admitted.

But it is more pleasant to the feelings of the sur. geon, who, after the greatest care to avoid the lens, and in the belief that he had done so, yet shortly discovers a rapidly growing cataract, to know that other explanations of its occurrence may be fortheoming; and these, I think, are not difficult to find.

To begin with, any one who will carefully examine the lenses in a series of apparently healthy senile eyes under a mydriatic will observe that in no small proportion of them he can discover peripheral striæ, not the normal striæ of the section of the lens visible by transmitted light in the eyes of many old people, but due to true cataractous change. In glaucomatous eyes these have been noticed in a still higher proportion.

In such eyes, or even in eyes without such lenticular change, it can scarcely be considered unreasonable to hold that the sudden alterations of conditions resulting from the emptying of the anterior chamber may have just as much effect in producing or in ripening cataract as has the well-known method of friction on it, directly or through the cornea. For those changes are very considerable: The zonular attachments are strained by the forward movement of the lens, and the lens may even come in contact with the cornea, while one must expect its nourishment to be seriously interfered with by the rapid passage from the eye of the fluid upon which its welfare depends, a fluid which also, as Nicati, Greef and others have shown, undergoes a change in composition upon the first opening of the anterior chamber.

\section{A CLINICAL NOTE ON THE USE OF PYOKTANIN.}

Read before the Section on Ophthalmology at the Forty-seventh Annual Meeting of the American Medical Association

BY H. B YOUNG, M.D.

BURLINGTON, IOWA.

My experience with pyoktanin probably differs little from that of your own in those suppurative troubles of the eye and ear for which it has been considerably lauded, i.e., occasionally very satisfactory, but more often disappointing, if not indeed aggravating. In fact after many disappointments I practically discarded it from my list of remedies as I have no doubt many have done.

About two years ago, however, after an unsuccessful attempt to treat a case that I had diagnosed as phlyctenular conjunctivitis, by the classical method, viz.: Pagenstecher's ointment, calomel dust and mydriatics locally, plus alterative and tonic medication internally, pyoktanin in $1-1000$ aqueous solution locally was accidentally suggested. To my surprise the case improved rapidly under it and was soon well. A month later the patient, an anemic girl of 15 years, returned with a fresh efflorescence. Again was the Pagenstecher's ointment and calomel dust distinctly irritating and the pyoktanin promptly effective. Following this at irregular intervals the efflorescence recurred and was promptly subdued by the pyoktanin. In the meantime a younger sister of this patient 
showed the same conditions, had the same treatment with the same result.

From this time all cases of conjunctivitis lymphatica were treated in this way and it was noticed that those were soonest benefited in which the efflorescence was marked by lachrymation and congestion of the palpebral conjunctiva, while the elevations at the limbus showed irregular outlines and little disposition to surface softening.

Why there should be this difference or why the pyoktanin should, in any case of this character, be as effective as the time honored mercurials I do not know.

Pharmaceutically the mercurials were as perfect as possible. The Pagenstecher's ointment I prepared by a method which could hardly be improved, as the product stands the test of inspection through a powerful lens. And the calomel was of the best quality, thoroughly dried and lightly dusted.

The pyoktanin was Merck's, dissolved in water which had been boiled thoroughly. A fresh solution was prepared when any change was noted in the color. DISCUSSION.

Dr. G. C. SAVAGE, Nashville-Just before leaving home I prescribed pyoktanin for three cases of phlyctenular conjunctivitis and as Dr. Price has just entered the room I should like to know from him what the result has been.

Dr. PRICE--The only one of the patients that has returned is doing well. It was a bad case and had shown no improvement under a week's use of the yellow oxid.

\section{FRANKLINIZATION AS A THERAPEUTIC MEASURE IN NEURASTHENIA.}

Read in the Section on Neurology and Medical Jurisprudence at the Forty-seventh Annual Meeting of the American Medical Association, held at Atlanta, Ga., May 5-8, 1896.

BY MARGARET A. CLEAVES, M.D.

Director of the New York Electro-Therapentic Clinic, Laboratory and Dispensary ; late Instructor in Electro-Therapeutics, New York Post

Graduate Medical Sehool; Fellow of the American Electro-
Therapeutic ssociation, Nember socicte Francaise D'liec. trotheravie, Fellow' of the New lork Academy of Medicine, Fellow of the Medical Society of the County of New rork. Fellow the American Medical Association, Hember the New York Electrical society. NEW YORK.

Looking at it from a purely scientific point of view the recognition under the head of neurasthenia of the many and varied expressions of nerve weakness is most unfortunate, and must have a tendency to hinder that investigation into the true nature of the underlying conditions which is absolutely essential to the advancement of the science of medicine. On the other hand this recognition has led to the establishment of excellent therapeutic methods, which, however, are so familiar as not to need enumeration here.

Among them Franklinization takes an important place, and although it is the oldest form of electric treatment, its value is not yet fully appreciated by the profession.

It is not within the scope of this paper to discuss the nature of neurasthenia, interesting as the subject is, suffice it to say that neurasthenia in an acquired form, may be regarded as primary or secondary; primary when due to the immediate effects of nervous overstrain or to a primary toxic disorder of nutritional processes, and secondary when consequent upon other diseases having a general pathologic diathesis with its peculiar and exhausting toxic influence.

and they break down even under slight overstrain, whether of work or mental anxiety. They lack in stability, and are incapable of ridding themselves of the results of physiologic activity. The toxicity thus induced plays an important role in the production of the varied manifestations of physical and mental discomfort to which the neurasthenic is subject.

The problem before us in the treatment of neurasthenia is to prevent undue waste of nervous substance from excessive fatigue as well as to eliminate the toxic influence of self-produced waste products. To this end whatever can be done to establish and maintain the highest nutritive activity is absolutely imperative.

Whether we regard the neurasthenic condition as due to exhaustion, starvation or poisoning of nerve centers its treatment by means of Franklinization is absolutely rational.

There is nothing of the occult about it, nor can the action of general Franklinization either by means of the convective or disruptive discharge be, in any sense, attributed to suggestion, hypnosis or to some subtle influence upon the nervous system. The same effects can not be reproduced by such methods.

The grand function of all electric treatment is to promote nutritive processes, and in general Franklin. ization there is no exception to the rule. On the contrary, by reason of its physical characteristics it is the most efficient of the varied manifestations of electric energy. The ease with which it can be used, requiring no preparation nor tedious detail in its administration as do otber currents, renders its application very acceptable to patients.

The influence of high frequency and high potential currents upon nutrition has been established by observations in physiologic laboratories and in clinical work.

In all neuroses there are abnormal chemic condi. tions of the tissues and aside from the influence of heredity, deficient constructive and deficient destructive metabolism are the two prominent factors therein. The need therefore in our therapeutics of a means which will influence a more nearly normal metabolism is imperative. The rationale of general Franklinization lies in its ability to set up processes resulting in the production of physiologic effects.

The increased activity of 'an organ is indicated by the increased amount of blood circulation. When an organ is completely inactive, as in the case of a paralyzed muscle or the peripheral end of a divided nerve, the amount of blood and the nutritive exchange of fluids diminish within these parts. Thus thrown out of activity they become pale and relaxed, and ultimately undergo fatty degeneration. If cells are imperfectly deprived of their detritus they do not appear to take up oxygen readily, consequently are not adequately nourished and undergo degenerative changes.

The average neurasthenic patient is not in a condition to take the active exercise necessary to bring muscular tissues into activity so as to profoundly influence circulatory changes. It is impossible to change the nutritional state of cells without a profound stimulating action. The general circulation in these conditions is impaired, while vascular changes in the nerrous system exist. These are of the nature of a cerebral hyperemia and probably hyperemia of The nutrition of nerve cells is primarily at fault, the spinal gray matter as well. The vaso-motor. 